\title{
Herbicide in drinking water linked to hormonal changes
}

More than 75 million pounds of the herbicide atrazine are applied annually to corn and other crops in the US and around the world. It is the most commonly detected pesticide contaminant in groundwater, surface water and rain, raising concerns about its safety.

Tyrone Hayes (University of California, Berkeley) and an international team of researchers reviewed the evidence linking exposure to atrazine to reproductive problems in animals, particularly amphibians and other creatures that encounter it in the water (J. Steroid Biochem. 127, 64-73; 2011). They found that when animals were exposed to even low ecological doses of the herbicide (e.g., $2.5 \mathrm{ppb}$ or below) during development, alterations of male reproductive tissues resulted, including decreases in testicular size, sperm production and androgen production. The 'feminization' of male gonads in many animals, sometimes to the point of complete sex reversal in genetic males, has caused shifts in sex ratios toward females in these populations. The extent of the shifts increases in proportion with increasing doses of atrazine.

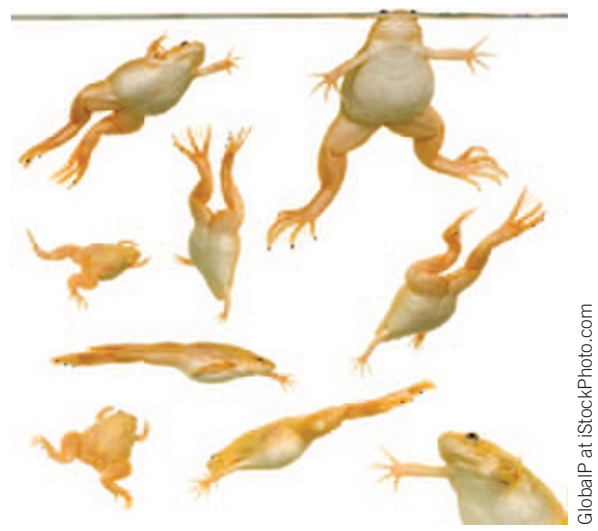

Epidemiological and laboratory evidence suggests that atrazine disrupts reproductive health and hormone secretion in female animals as well. To study whether similar effects are present in humans exposed to atrazine, Lori A. Cragin and colleagues at Colorado State University (Fort Collins), together with their collaborators, compared women in Illinois, where atrazine was detected in the water supply at concentrations of $0.7 \mathrm{ppb}$, to women in Vermont, where atrazine is not commonly used as a pesticide (Environ. Res. 111, 1293-1301; 2011).

Women who drank water containing atrazine had significantly lower levels of estrogen, which were inversely related to the estimated dose of atrazine that they ingested. They also were five times more likely to report irregular periods than women in Vermont, with the likelihood even greater for women who said they drank more than two cups of local tap water daily. The authors of the study considered the effects of lifestyle factors, such as physical activity, weight and diet, but these were not significant. These results are consistent with some studies that suggest that atrazine may block the production of estrogen in the female body. These hormonal changes have been associated with increased risk of osteoporosis, diabetes, heart disease and some cancers.

More than 150 new studies since 2003 have uncovered potential health effects of atrazine. In light of the safety concerns raised by these studies, scientists are calling for increased regulation of herbicides and a discontinuation of atrazine's use.

\section{Kara Rosania}

\section{CLEFT LIP CORRECTED GENETICALIY IN MOUSE}

Cleft lip with or without cleft palate $(\mathrm{CL} / \mathrm{P})$ is the most frequent facial birth defect in humans, with an incidence of 1 in 700 live births. Current treatment requires multiple cycles of surgery, speech therapy and orthodontics, placing a great burden on those born with the defect.

$\mathrm{CL} / \mathrm{P}$ is caused by the disruption of the genetic programs that guide facial development. In a new study, genetic methods were used for the first time to successfully repair cleft lip in mouse embryos. By mutating three Pbx genes in the embryonic cell layer that gives rise to the lip and nose, Licia Selleri and her team at Weill Medical College of Cornell University (New York, NY) created the first multi-gene transgenic mouse model for CL/P (Dev. Cell 21, 627-641; 2011).

$\mathrm{Pbx}$ genes are highly expressed in the area where the developing layers of facial tissue fuse together. Pbx proteins coordinate cellular activities crucial for facial development by regulating a chain of signaling molecules that have been implicated in $\mathrm{CL} / \mathrm{P}$, including Wnt, best known for their roles in development. This signaling pathway is conserved across mammalian species. Only the mouse lines lacking multiple $\mathrm{Pbx}$ genes simultaneously in the facial tissue layer exhibited complete $\mathrm{CL} / \mathrm{P}$. This is consistent with human $\mathrm{CL} / \mathrm{P}$, which is caused by multiple concomitant gene mutations.

The mouse embryos with multiple $P b x$ mutations had reduced or absent Wnt activity and disrupted Wnt signaling, suggesting that $\mathrm{Pbx}$-directed Wnt signaling is required for proper facial development. In fact, in the embryos with compound $P b x$ mutations, restoring the Wnt signaling pathway was sufficient to correct the defects: the cleft lips in all of these animals completely disappeared. Selleri explained, "To my knowledge, this is the first time that anyone has corrected this defect in embryos, and we really show here that Wnt is a critical factor. This is a provocative result because it opens a completely new avenue of strategies for tissue repair."

In follow-up studies, the researchers plan to supply Wnt molecules directly, or implant tissues that would deliver Wnt molecules, to $P b x$-mutated mouse embryos placed within a uterus-like environment. This approach of delivering Wnt signals directly to the uterus would be more realistic for implementation in humans. If the Wnt treatment is sufficient to correct or even prevent the facial developmental abnormalities, it may provide a means of correcting CL/P before it even occurs.

Kara Rosania 